University of Wollongong

Research Online

Faculty of Engineering and Information

Faculty of Engineering and Information

Sciences - Papers: Part B

Sciences

2010

On Real-time Whole-body Human to Humanoid Motion Transfer

Francisco-Javier Montecillo-Puente

LAAS-CNRS Toulouse France

Manish Sreenivasa

University of Wollongong, manishs@uow.edu.au

Jean-Paul Laumond

LAAS-CNRS Toulouse France

Follow this and additional works at: https://ro.uow.edu.au/eispapers1

Part of the Engineering Commons, and the Science and Technology Studies Commons

Research Online is the open access institutional repository for the University of Wollongong. For further information contact the UOW Library: research-pubs@uow.edu.au 


\title{
On Real-time Whole-body Human to Humanoid Motion Transfer
}

\author{
Abstract \\ We present a framework for online imitation of human motion by the humanoid robot HRP-2. We \\ introduce a representation of human motion, the humanoid-Normalized model, and a Center of Mass \\ (CoM) anticipation model to prepare the robot in case the human lifts his/her foot. Our proposed motion \\ representation codifies operational space and geometric information. Whole body robot motion is \\ computed using a task-based prioritized inverse kinematics solver. By setting the human motion model as \\ the target, and giving the maintenance of robot CoM a high priority, we can achieve a large range of \\ motion imitation. We present two scenarios of motion imitation, first where the humanoid mimics a \\ dancing motion of the human, and second where it balances on one foot. Our results show that we can \\ effectively transfer a large range of motion from the human to the humanoid. We also evaluate the \\ tracking errors between the original and imitated motion, and consider the restrictions on the range of \\ trans ferable human motions using this approach.

\section{Keywords} \\ real-time, motion, humanoid, human, whole-body, transfer \\ Disciplines \\ Engineering | Science and Technology Studies

\section{Publication Details} \\ Montecillo-Puente, F., Sreenivasa, M. N. \& Laumond, J. (2010). On Real-time Whole-body Human to \\ Humanoid Motion Transfer. In J. Filipe, J. A. Cetto \& J. Ferrier (Eds.), Proceedings of the 7th International \\ Conference on Informatics in Control, Automation and Robotics - Volume 2 (pp. 22-31). SciTePress.
}




\title{
ON REAL-TIME WHOLE-BODY HUMAN TO HUMANOID MOTION TRANSFER
}

\author{
Francisco-Javier Montecillo-Puente, Manish N. Sreenivasa and Jean-Paul Laumond \\ CNRS, LAAS, 7 avenue du colonel Roche, F-31077 Toulouse, France \\ Université de Toulouse, UPS, INSA, INP, ISAE, LAAS, F-31077 Toulouse, France \\ $\{$ fmonteci, manu, jpl\}@laas.fr
}

Keywords: Humanoids, HRP2, Human motion, Inverse kinematics, Neuroscience.

\begin{abstract}
We present a framework for online imitation of human motion by the humanoid robot HRP-2. We introduce a representation of human motion, the Humanoid-Normalized model, and a Center of Mass (CoM) anticipation model to prepare the robot in case the human lifts his/her foot. Our proposed motion representation codifies operational space and geometric information. Whole body robot motion is computed using a task-based prioritized inverse kinematics solver. By setting the human motion model as the target, and giving the maintenance of robot CoM a high priority, we can achieve a large range of motion imitation. We present two scenarios of motion imitation, first where the humanoid mimics a dancing motion of the human, and second where it balances on one foot. Our results show that we can effectively transfer a large range of motion from the human to the humanoid. We also evaluate the tracking errors between the original and imitated motion, and consider the restrictions on the range of transferable human motions using this approach.
\end{abstract}

\section{INTRODUCTION}

Since the inception of humanoid robots several approaches have been developed, aiming at enabling autonomous and intelligent behavior of the robot. The general goal behind this idea is to develop humanoids that, in the natural environment of the humans and being among humans, can exhibit complex behavior. The humanoid and the human share a common anthropomorphic structure, and recent studies have exploited this to directly transfer motion from humans to humanoids. However, several obstacles imped the direct transfer of motion like technological incompatibility of the humanoid robot, fragility, slow (relative to human) motion speed, as well as a limited range of motion.

There are several ways to approach the motion transfer problem. In computer graphics, studies have looked at "motion retargeting", where human motion is transferred from one virtual actor to another (Multon et al., 2008)(Chois and Ko, 2000). Machine learning approaches have also been developed to generate humanoid motion by observing and learning from a human teacher (Schaal et al., 2003) (Shon et al., 2005) (Takano et al., 2007). Another method is to optimize the recorded human motion while considering humanoid kinematics and dynamics (Suleiman

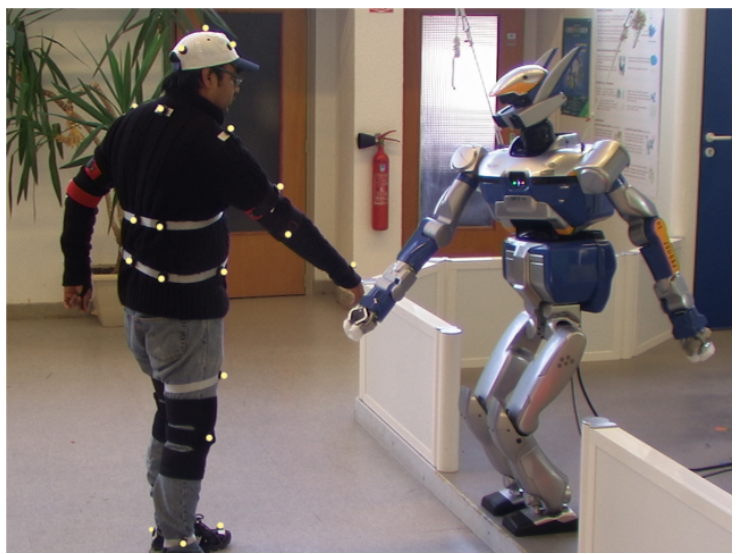

Figure 1: Picture of a human performer extending a handshake and the humanoid robot HRP-2 imitating the gesture. HRP-2 is a 30 Dof, $58 \mathrm{~kg}, 1.54 \mathrm{~m}$ tall humanoid robot manufactured by Kawada Industries, Japan. The human motion was tracked using reflective motion markers and transferred in real-time to the humanoid.

et al., 2008) (Ruchanurucks et al., 2006). For our purpose we broadly classify motion imitation studies into offline and online methods. Offline methods have the advantage of having ample processing time and hence the human motion can be modified to fit the humanoid's limitations. While this allows for gen- 


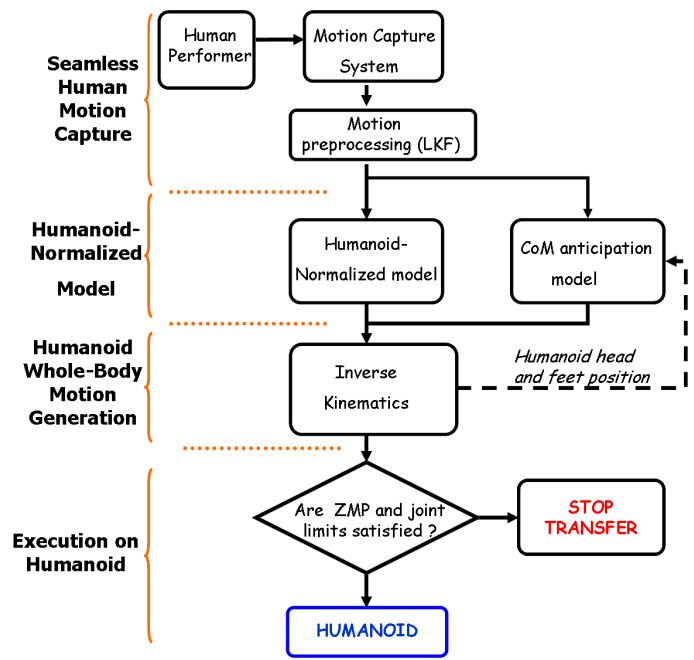

Figure 2: Organization of the algorithm to enable real-time motion transfer from the human performer to the humanoid robot.

eration of smooth and optimized motion of the humanoid, these solutions can rarely be applied to online motion transfer, often due to computational delays during the optimization stage. Thus, online transfer of human motion to a humanoid robot provides a very different set of challenges compared to its offline counterpart, and has been the focus of recent research (Dariush et al., 2008a) (Dariush et al., 2008b) (Yamane and Hodgins, 2009).

We explore this aspect of real-time humanhumanoid motion transfer by developing a framework that allows us to transfer a large range of human motions to the humanoid, including balancing on one foot. The challenges in this task include reliably recording human motion, morphing the human data such that it can be applied on a humanoid of a different size and joint structure, and, generating the resulting humanoid motion in real-time while maintaining balance.

\subsection{Literature Review}

\subsubsection{Offline Motion Transfer}

First we examine the work by Nakaoka et. al in 2005 (Nakaoka et al., 2005a), that enabled the humanoid robot HRP2 to execute the famous and visually striking, traditional Aizu-Bandaisan Japanese dance. Upper body motion for the HRP1s robot was generated using motion capture markers on human dancers and inverse kinematics. The leg motion of the dancers was analyzed and extracted as motion primitives (Nakaoka et al., 2005b). The upper and lower body motions were then coupled and modified such that the robot motion satisfied the dynamic stability criteria, the Zero Moment Point (ZMP) (Vukobratovic and Stepanenko, 1972). This method was implemented offline and required several runs of the analytical process to reach a viable solution. However, this method is not be easily applicable to a range of motions due to the extensive tuning and re-calculation required. As a more general framework, Ude et. al proposed to solve a large scale optimization problem to generate joint trajectories for a DB robot (Ude et al., 2004). Joint angle trajectories of human motion were computed by embedding a scalable kinematic structure to the human body motion. This was also solved as an offline process because of the optimization computational load. Robot balance was not taken into consideration.

In the study by Ruchanurucks et al. (Ruchanurucks et al., 2006), a non-linear optimization process was solved subject to joint limits, autocollision, velocity limits and force limits constraints. To increase the convergence speed they parametrized the motion by B-splines. In Suleiman et. al (Suleiman et al., 2008), first the joint motion data was scaled into the humanoid robot's joints. Then, an optimization problem was solved to fit this motion to the robot structure and its physical limits, keeping in mind the analytical gradient of the dynamic model. Among other approaches, studies have also used machine learning algorithms to imitate human motion (Shon et al., 2005). The idea here was to generate a low dimensional latent space to map a model variable from the robot motion to human motion, and vice-versa. In order to generate the final stable motion, extensive training was required using pairs of human and robot motion.

In most of these studies the humanoid is constrained to maintain both its feet on the ground. Even in the approaches that allowed feet motion, for example (Suleiman et al., 2008) and (Ruchanurucks et al., 2006), the timing of the foot lift-off has to be predefined which results in a rigid range of motion.

\subsubsection{Online Methods}

In the context of online transfer of human motion to humanoid robots or even virtual avatars in animation, the means used to capture human motion plays a very important role. One way is the use of motion capture technology. These systems represent human motion by directly tracking the position of infra-red markers attached to the human body or attaching a skeleton to these markers. However, some recent studies have also implemented markerless tracking where human motion is reconstructed by using video cameras.

First, we consider the study by Dariush et al. (Dariush et al., 2008b), where the authors developed a 
methodology to retarget human motion data to the humanoid robot ASIMO. Human motion was captured using a markerless pose tracking system. Here upper body motion was considered by mapping the cartesian positions of the waist, wrists, elbows, shoulder and neck. The corresponding joint motion on the humanoid was calculated using inverse kinematics, while respecting the individual joint limits. In this case, a seperate balance controller was used to move the legs in order to compensate for the retargeted motion of the upper body. In a later study, the authors used a learning approach to pre-generate knowledge about a number of human postures (Dariush et al., 2008a). During the actual motion retargeting, head and torso motion was monitored and the template closest from the ones learned was assigned. The arms were analyzed as 3D blobs and their position estimated. From this data the 3D features for head, shoulder, waist, elbows and wrists were localized. Using inverse kinematics and the balance controller, the motion was then played on the humanoid robot.

Using a different approach, Yamane et al. (Yamane and Hodgins, 2009), simultaneously tracked motion capture data using a balance controller and a tracking controller. Tha balance controller was a linear quadratic regulator designed for an inverted pendulum model. The tracking controller computed joint torques to minimize the difference from the desired human capture data while considering full-body dynamics. The resulting motion was retargeted on the humanoid in simulation.

\subsection{Our Contribution}

We present an alternative way to transfer human motion data to our humanoid robot HRP2.

- Taking inspiration from computer animation studies we extended the idea of the normalized skeleton (Multon et al., 2008) and developed a "Humanoid-Normalized model" on which filtered motion capture data can be retargeted online. To do this we first devise a method to reliably record human motion by using Kalman filters to fill gaps (due to occlusions) in capture data. Rather than directly using cartesian positions like in (Dariush et al., 2008a) (Dariush et al., 2008b), or joint angles (Yamane and Hodgins, 2009), we also encode the orientation of the postures by attaching virtual planes to sets of human motion marker points. These virtual planes define the orientation of the important joints in the human, like the head, chest, arms and waist. We propose a Humanoid-Normalized model that consists of a combination of the positions of the extremities and the normals to the virtual planes. The motion of this model is used to drive the humanoid robot via a task based inverse kinematics solver.

- In order to achieve single foot support phases we also introduce the original idea of an anticipation model, motivated by results in human neuroscience. This model serves the purpose of preparing the humanoid to stand on one foot by taking into account previous motion of the human head, and the humanoid's Center of Mass (CoM). Figure 2 shows the organization of the overall algorithm.

We evaluate the results from our experiments and compare them to those reported in literature. In the following sections we describe in detail 1) The capture of human motion capture data, 2) Application to the Humanoid-Normalized model, 3) Generating humanoid motion using inverse kinematics 4) The CoM Anticipation model 5) Experiments on our humanoid robot, HRP2 and finally 6) Discussion of our results as well as future perspectives.

\section{SEAMLESS HUMAN MOTION CAPTURE}

Human motion was recorded in a tracking hall equipped with 10 infra-red tracking cameras (MotionAnalysis, CA, USA). The system is capable of tracking the position of markers within a $5 \times 5 \mathrm{~m}$ space within an accuracy of $1 \mathrm{~mm}$, at a data rate of $100 \mathrm{~Hz}$. The human to be tracked wore 41 reflective markers firmly attached to their body using velcro straps, or tape (see Figure 1 and 3). In some cases there was the possibility of loss of marker data due to selfocclusions. This is very detrimental to the imitation algorithm since the humanoid model depends on continous human motion data. To solve this, we implemented a linear Kalman filter that estimates the position of markers that are lost for short durations of time (less than 0.5 seconds). If a marker is lost for periods longer than 0.5 seconds, we assume the model to be unrecoverable and initiate an emergency stop. The latency between capture of marker data and the kalman filtered data is about $30 \mathrm{~ms}$. This filtered data is then applied to the Humanoid-Normalized model. 


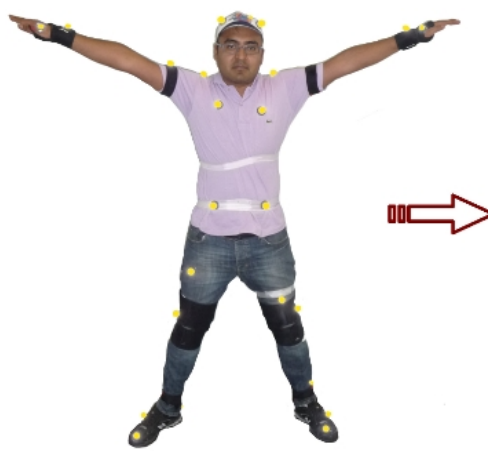

HUMAN

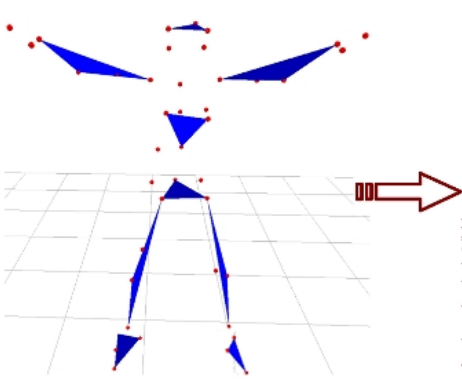

MODEL

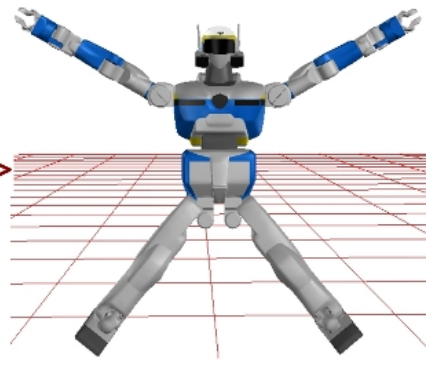

HUMANOID

Figure 3: Human $\rightarrow$ Humanoid Normalized Model $\rightarrow$ Humanoid. Motion capture position data from the human is transferred to the normalized model and associated with the planes and humanoid joints. The motion of these planes and joints drives the humanoid motion.

\section{HUMANOID-NORMALIZED MODEL}

\subsection{Why a Normalized Model?}

Multon et al. (Multon et al., 2008) proposed the use of a normalized skeleton, which acts as an intermediate entity to map motion between two different digital actors. The generation and adaptation of motion was done by assuming that there exists planes formed by the arms and legs. The normalized skeleton was represented by normalized segments, limbs of variable lengths and the spine. Humans come in different sizes and shapes, and most adults are bigger than our humanoid HRP2. HRP2 also has two additional DoF's in its chest (pitch and yaw) and this requires special treatment unlike that for the humanoid ASIMO, used in (Dariush et al., 2008a), where the torso is one single joint. There is thus a need to extract the relevant characteristics of human motion, and define it in a form that is suitable for application on humanoids. We extended the idea presented by Multon and colleagues by defining planes for not only the arms, but also the head, chest and waist. The planes are attached to the human body in such a way that they can be directly used to define geometric tasks in the prioritized inverse kinematics solver. A combination of the orientation of these planes, and the position of the extremities (head and wrists) form the HumanoidNormalized model (henceforth HN Model).

\subsection{Components of Humanoid-normalized Model}

The physical model of the upper body was built from the cartesian positions of 22 markers on the human
Figure 3. Before the model is computed we scale the marker positions according to a modified version of a standard scaling algorithm (Boulic et al., 2009). First, we start with the head. Three markers on the head were used to form the head virtual plane. The center of these markers were considered in the $\mathrm{HN}$ model. The normal to the plane defined by these markers was computed. In the HN model we represent the orientation of the head as the orthogonal to this normal vector. For the head, the plane normal is computed as,

where,

$$
N_{\text {head }}=V_{0} \times V_{1}
$$

$$
\begin{aligned}
& V_{0}=\frac{p_{0}-p_{1}}{\left\|p_{0}-p_{1}\right\|} \\
& V_{1}=\frac{p_{0}-p_{2}}{\left\|p_{0}-p_{2}\right\|}
\end{aligned}
$$

$p_{0}, p_{1}, p_{2}$ are the markers associated to the head virtual plane.

Similarly, chest and waist virtual planes were constructed using the relevant markers such that their normals were approximately in the sagittal direction (see Figure 3 for illustration of markers used). Virtual planes for the arms were constructed using markers on the shoulder, elbow and wrist. Instead of using the positions of all these markers, the arm posture is represented by the normal to this plane and the wrist position. We chose this representation because of the difficulty to directly map the cartesian position of all these markers to the robot structure. In addition to upper body characteristics, we also included the position of the feet in the HN model.

Thus, overall the Humanoid-Normalized model is expressed by the following set of geometric properties:

$$
\left[P_{h}, V_{h}, N_{c}, V_{w}, P_{l h}, N_{l a}, P_{r h}, N_{r a}, P_{l f}, P_{r f}\right]
$$


where $P_{h}$ is a point representing the position of the head, $V_{h}$ is a vector representing the orientation of the head, $N_{c}$ is a vector representing the normal of the chest plane, $V_{w}$ is a vector representing the orientation of the waist, $P_{l h}$ is a point representing the position of the left hand, $N_{l a}$ is a vector representing the normal to the left arm plane, $P_{r h}$ is a point representing the position of the right hand, $N_{r a}$ is a vector representing the normal to the right arm plane, $P_{l f}$ is a point representing the position of the left foot, $P_{r f}$ is a point representing the position of the right foot.

\section{HUMANOID INVERSE KINEMATICS}

The HN model explained in the previous section allows us to retarget human motion into a form suitable for transfer onto the humanoid. To generate motion of the humanoid we used a task-based inverse kinematics solver (Nakamura, 1991).

\subsection{Prioritized Inverse Kinematics}

Forward kinematics expresses the relationship between the variation of the joint parameters $\delta q$ and the corresponding displacement $\delta x$ in the operational space (Nakamura, 1991). This is given by,

$$
\delta x=J \delta q
$$

where $J$ is the $m \times n$ jacobian matrix, $m$ being the dimension of the task, and, $n$ is the number of degrees of freedom. The inverse kinematics model determines the joint variation that produces an expected displacement, which is obtained by solving the system in Eq. 3 . However, for the humanoid robot we have $m<n$, thus this linear system is under-constrained. In this case, all the solutions of the system can be written as:

$$
\delta q=J^{\#} \delta q+\left(I-J^{\#} J\right) z
$$

where $J^{\#}=J^{T}\left(J J^{T}\right)^{-1}$ is the pseudo inverse of $J, I$ is the $n \times n$ identity matrix, $\left(I-J^{\#} J\right)$ is the null-space projector of $J$, and $z$ is an $n$-dimensional arbitrary vector. The first term on the right side of equation 4 is a generic solution and $z$ in the second term can be used to satisfy additional constraints without modifying $\delta x$.

The generic expressions for solving $n$ tasks with descending order of priorities are (Siciliano and Slotine, 1991):

$$
\begin{aligned}
N_{0} & =I \\
\hat{J_{i}} & =J_{i} N_{i-1} \\
N_{i} & =I-\hat{J_{i}^{\#}} \hat{J}_{i}
\end{aligned}
$$

$$
\delta q_{i+1}=\hat{J}_{i+1}^{\#}\left(\delta x_{i+1}-J_{i+1} \delta q_{i}\right)
$$

where $N_{i}$ is the projector associated with the $i^{t h}$ task, $J_{i}$ is the jacobian of the $i^{t h}$ task, $I$ is the $n \times n$ identity matrix. The update $\delta q_{i}$ is iteratively computed for all tasks.

\subsection{Whole-body Motion Generation}

The position for each joint is generated from a prioritized stack of tasks (Yoshida et al., 2006), which is solved using the inverse kinematics formulation expressed above. Each property of the HN model representation is used as the target input for the tasks. Our task stack was defined as (in decreasing priority):

1. Homogenous transformation task for each feet, i.e. both position and orientation are fixed,

2. Position task for Center of Mass (CoM) projection (X and Y positions),

3. Position task for the head,

4. Homogenous transformation task for the left wrist,

5. Homogenous transformation task for the right wrist,

6. Orientation vector task for the chest,

7. Orientation vector task for the waist,

8. Orientation vector task for the head.

We use four kinds of tasks: position task, orientation vector task, homogenous transformation task and a CoM task. As examples, we define in more detail the task construction for the head and the arms. The CoM task is detailed in the next section. The position task of the head $f_{h}(\theta)$ is defined as,

$$
f_{h}(\theta)=P_{h}^{t}-P_{h}(\theta)
$$

where $P_{h}^{t}$ is the target of the task given by the position of the head in the $\mathrm{HN}$ model representation. $P_{h}(\theta)$ is the position of the humanoid head expressed as a function of the robot dof's $\theta$.

For the orientation vector task of the head $f_{h}(\theta)$ we have

$$
f_{h}(\theta)=V_{h}^{t} \times V_{h}(\theta)
$$

where $V_{h}^{t}$ is the target head direction, which is given by the head orientation vector in the $\mathrm{HN}$ model. And $V_{h}(\theta)$ is the corresponding vector of the humanoid head, as a function of the robot dof's $\theta$. The orientation vector tasks for the chest and waist are defined in a similar way.

For each arm a homogeneous transformation task is constructed for the wrist joint. The target transformation is constructed from two properties, wrists 

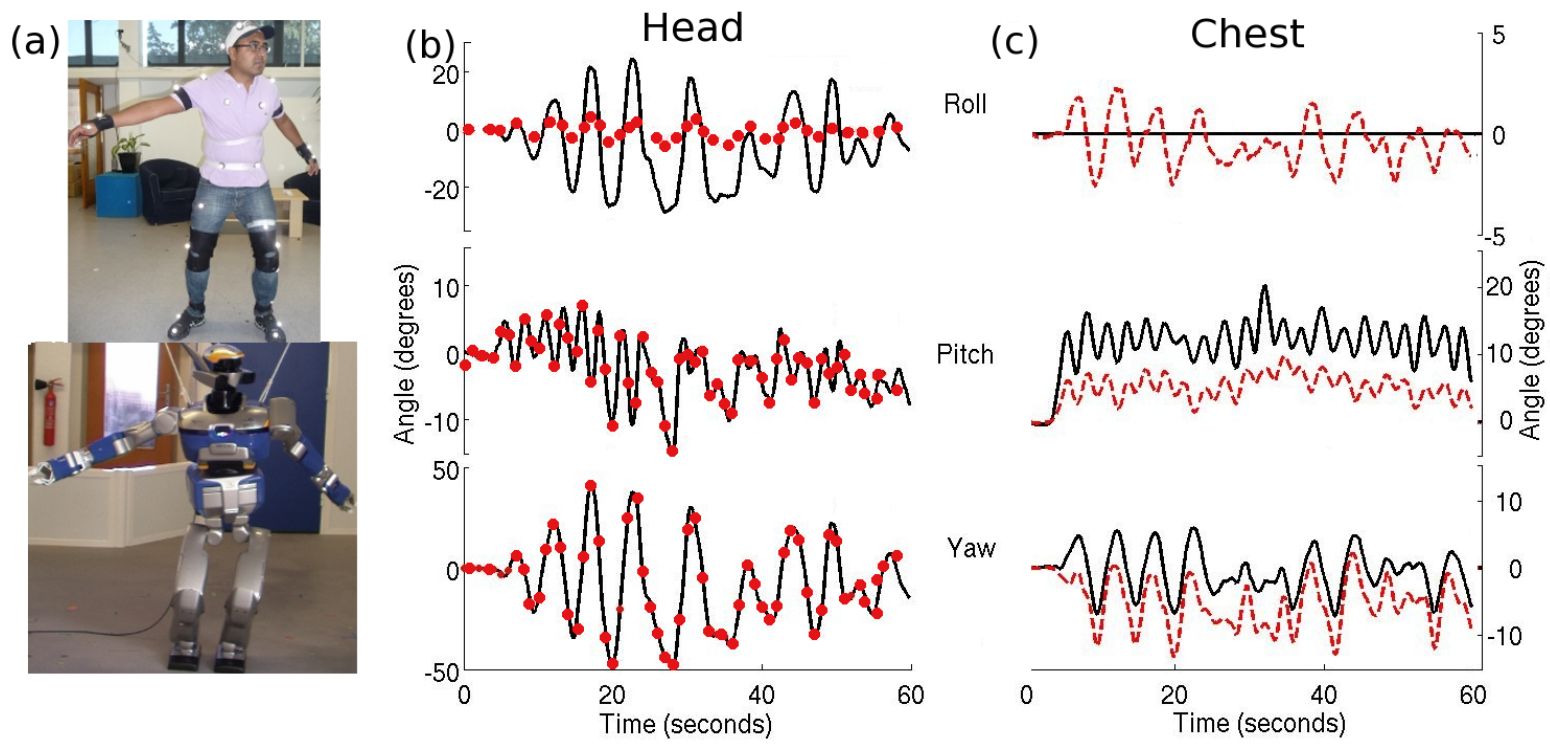

Figure 4: Scenario 1. (a) Snapshot of human dancing and its imitation by HRP2 (b) Roll, pitch and yaw angles of the head joint during the dancing motion. Solid black line indicates the angles of the HN model, this was the target the humanoid had to follow. Red circles indicate the corresponding angular value on HRP2 (c) Chest angles of the HN model (solid black line) and the corresponding angles on the humanoid (dashed red line).

position and the normal to the HN model's arm plane. The target rotation matrix in the homogenous transformation is computed as,

$$
R^{t}=\left[\begin{array}{lll}
N_{\text {arm }} \times V & N_{\text {arm }} V
\end{array}\right]
$$

where $N_{\text {arm }}$ is the normal of the left or right arm plane, and $V$ is a unit vector connecting the elbow and wrist markers. It should be noted that the $N_{\text {arm }}$ is parallel to the axes of the humanoid elbow joint.

\section{COM ANTICIPATION MODEL}

The CoM of a humanoid robot is a vital indicator to its stability. In order to remain statically stable, the projection of CoM on the floor should remain within the support polygon defined by the two feet of the humanoid. If the human performer were to lift his/her foot, the CoM of the humanoid robot would have to be shifted in advance towards the other foot in order to maintain balance. In order to know when this shift is required, we take inspiration from results in human neuroscience research. Studies have reported strategies by which motion of the CoM in humans can be related to foot placement and hip orientation (Patla et al., 1999), (Vallis and McFadyen, 2005).

To manipulate the projection of the humanoid $\mathrm{CoM}$ on the floor we constrain it to track a target. The target position is computed depending on the current stance of the HN model, i.e. Double Support (DS) or Single Support (SS). The transition of stance from single to double support is detected using the position and velocity of the feet. When either of these measures exceed a pre-determined threshold a change of stance is said to have occured. For the motion of the CoM the target is computed as:

$$
\operatorname{CoM}_{i}=\left\{\begin{aligned}
\operatorname{CoM}_{i-1}+\alpha\left(V_{\text {head }} \cdot V_{\text {feet }}\right) V_{\text {feet }} & \text { if DS } \\
p_{\text {foot }}+\beta V_{\text {head }} & \text { if SS }
\end{aligned}\right.
$$

where,

$\operatorname{CoM}_{i}=\mathrm{CoM} \mathrm{X}$ and $\mathrm{Y}$ positions at time step $i$,

$V_{\text {head }}=$ HN Model head 2D velocity vector,

$V_{\text {feet }}=$ Unity vector across robot's feet,

$p_{\text {foot }}=$ Humanoid support foot $\mathrm{X}$ and $\mathrm{Y}$ positions,

$\alpha, \beta$ are constants.

\section{IMPLEMENTATION OF ONLINE HUMAN-HUMANOID MOTION TRANSFER}

Our framework was implemented using the software achitecture Genom, (Fleury et al., 1997). Mainly, we have four modules to establish communication from the motion capture system to the HRP2 robot interface. First, we have a motion capture server whose function is to send motion data to the network via UDP protocol. These data are filtered as described 


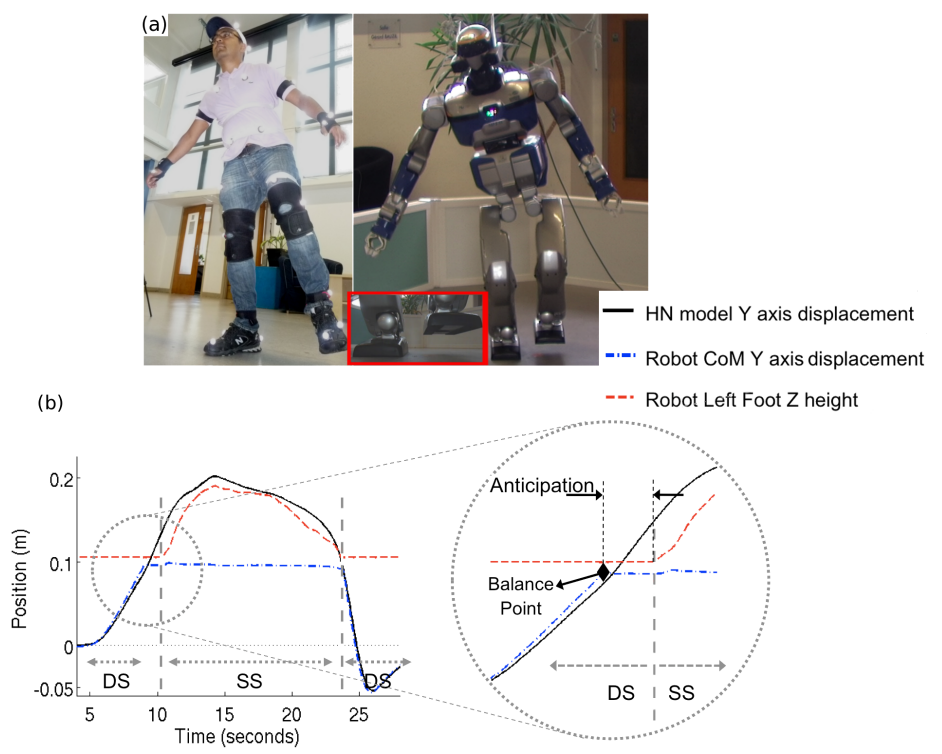

Figure 5: Scenario 2. (a) Picture of humanoid imitating the human lifting his foot. (b) Sideways displacement of the HN model head (solid black line), and the vertical height of the humanoid's lifting foot (dashed red line). Also shown is the $\mathrm{Y}$ displacement of the humanoid's CoM (dash-dot blue line). Zoom inset shows a magnified view of the anticipation phase. The anticipation occurs between the 'Balance point' and the time when the humanoid lifts its foot.

in section 2. A second Genom module reads the seamless motion data and computes the robot motion. This module implements the HN-normalized model, the CoM anticipation model and the prioritized inverse kinematics solver. Finally, via a plugin we send robot motion data from the motion generation Genom-module to the HRP2 interface control panel.

We present two scenarios that illustrate the capabilities of our algorithm. In the first scenario we assume the robot's feet to be fixed and imitate the motion of a human performer executing a slow dance with the upper body, including bending of the knees and ankles. In the second scenario, the robot is allowed to lift-off with one of its feet and balance on the other foot. This was chosen to illustrate the anticipation model which prepares the humanoid for balancing on one foot. The parameters used for the CoM anticipation model were, $\alpha=0.12, \beta=$ 0.01. All computations were run on an Intel Core 2 CPU 6400 @2.13GHz, with 2GB of RAM memory. At each solution step we required $\sim 30 \mathrm{~ms}$ to build the Humanoid-Normalized model and to solve the stack of tasks using a damped inverse kinematics solver. The video of the results can be accessed at http://homepages.laas.fr/manu/videos/motionImitation.mp4

\subsection{Dancing}

The human performer was asked to perform a simple dance without stepping or sliding his feet. Figure 4-a shows the posture of the human and the humanoid in the middle of the dance. The motion computed by the algorithm was smooth, without joint position or velocity limit violations, and was quasi-statically stable. Figure 4-b \& c show the roll, pitch and yaw angles of the head and chest of the HN model and those of the humanoid robot. Despite the low priority given to the head orientation task, we see that the yaw and pitch angles were matched very closely, while roll angle of the humanoid was much lesser than the HN model. This was because the yaw and pitch axis are directly available on HRP2 (independent of the other joints), however, the humanoid does not have a roll axis for the head joint. The roll variation seen in Figure 4-b was due to the movement of the whole body. Chest roll of the HN model was not considered, but to account for the movement of the rest of the body we see an induced roll component on HRP2. The pitch and yaw angles of the humanoid's chest followed the $\mathrm{HN}$ model less closely due to the lower priority of this task. Since the arms and the head are connected to the chest, and their respective tasks have a higher priority, the chest joint has a reduced degree of mobility.

\subsection{Foot Lift}

In this scenario the human performer shifted his weight onto one leg and maintained his balance for a few seconds before slowly returning to rest on both feet. Figure 5-a shows the human and the humanoid balancing on one foot (SS stance). The motion of the 
Table 1: Mean RMS error between HN model and humanoid. Values in brackets denote the mean RMS error in $\mathrm{X}, \mathrm{Y}$ and $\mathrm{Z}$ positions for wrist positions, and roll (R), pitch (P) and yaw (Y) for head, chest and waist orientations.

\begin{tabular}{|c|c|c|}
\hline Property & $\begin{array}{c}\text { Mean RMS } \\
\text { position (m) }\end{array}$ & $\begin{array}{c}\text { Mean RMS } \\
\text { orientation (deg) }\end{array}$ \\
\hline \hline CoM & $\sim 0$ & - \\
\hline Head & $\sim 0$ & 4.09 \\
& (R: 11.8 P: 0.22 Y: 0.26) \\
\hline Left wrist & 0.02 & - \\
& (X: 0.013 Y: 0.02 Z: 0.3) & - \\
\hline Right wrist & 0.05 & 4.2 \\
& (X:0.05 Y:0.017 Z: 0.08) & \\
\hline Chest & - & (R: 1.1 P: 6.7 Y: 4.8) \\
\hline Waist & - & 6.4 \\
& & (R: 1.1 P: 4.62 Y: 0.52) \\
\hline
\end{tabular}

head in the HN model, and the vertical position of the foot of the humanoid is plotted in Figure 5-b. We observe that the head shifts towards the support foot (right foot) before the lifting the other foot (Figure 5-b). The sideways displacement of the head reaches the Y position of the support foot about $1 \mathrm{~s}$ before foot lift. Before reaching this point, the CoM projection was derived according to Eq. 7 (DS stance). Once the head reaches the support foot, the CoM is maintained at this position (referred to as "Balance Point" in Figure 5-b). After this point, the behavior of the $\mathrm{CoM}$ is dictated by a different relation (SS stance in Eq. 7). It should be noted that for slow head motion, the projection of the CoM and the head position coincide (a small offset can be seen in the zoom inset in Figure 5-b)).

\section{DISCUSSION}

Among studies that have looked at human-humanoid motion retargeting it is generally difficult to define a single metric reflecting the quality of motion transfer. This can be because of several reasons. The physical structure of humanoids can vary quite significantly (size, number of DoFs, range of motion etc). Moreover, defining a mathematical term to the rather abstract idea of "good" motion imitation is not clear. Dariush and colleagues, formulated a measure for "tracking error" to express the effectiveness of their algorithm. We employ a similar strategy and additionally investigate the limitations of our approach vis-à-vis dynamic stability of the humanoid.

\subsection{Quality of Motion Imitation}

Quality of motion imitation was quantified by measuring the root mean square error between the target (HN model) and the humanoid robot. Table 1 lists the relevant parameters and the errors. The position of the CoM and head were tracked almost prefectly. This was because both these tasks had a very high priority. Comparitively, head orientation which had a lower priority had a mean error of $4 \mathrm{deg}$. But it should be noted that most of this error was because of the roll angle (HRP2 does not have a head roll axis). The right wrist position error was slightly larger that the left wrist. This can be attributed to the fact that left wrist task came before the right wrist task in the priority list. Thus, once the left wrist position and orientation was fixed, it became more difficult for the right wrist to reach exactly its target transformation. Comparing across studies, Dariush et al. 2008b, reported an error of about $0.02 \mathrm{~m}$ in tracking the wrist position while assigning them to a "medium priority group". In our case the head was the highest priority, and hence a low error, while the hands were low priority, hence the larger error. Chest and waist orientation were lower in the priority list and hence show larger errors in orientation than the other joints. Overall, these results show that we were able to retarget a large part of the motion of the human onto the humanoid.

\subsection{Limitations}

We analysed the limitations of our imitation system, and those of our humanoid, by setting up the following test. We attached the marker set on the performer and he was asked to move his right hand in an up-and-down motion at different speeds. After transferring the motion to the humanoid, we observed the shift of the Zero Moment Point (ZMP) of the humanoid for the different human hand speeds. For the up and down motion, we detect the maximum and minimum values of the ZMP components, and check if it is inside the supporting polygon. We found that the humanoid became unstable when the hand speed was higher than $1 \mathrm{~m} / \mathrm{s}$ (Figure 6). This example illustrates the limitations of using inverse kinematics without considering, simultaneously, the dynamic stability of the humanoid. To truly imitate both the kinematics and dynamics of the human motion, it would be important to take both of these into account during the modeling stage itself. For example, using a dynamical model (exact or simplified) at the motion planning stage could be a useful in this regard.

Kanehiro et al. (Kanehiro et al., 2008b), devised a 
(a)

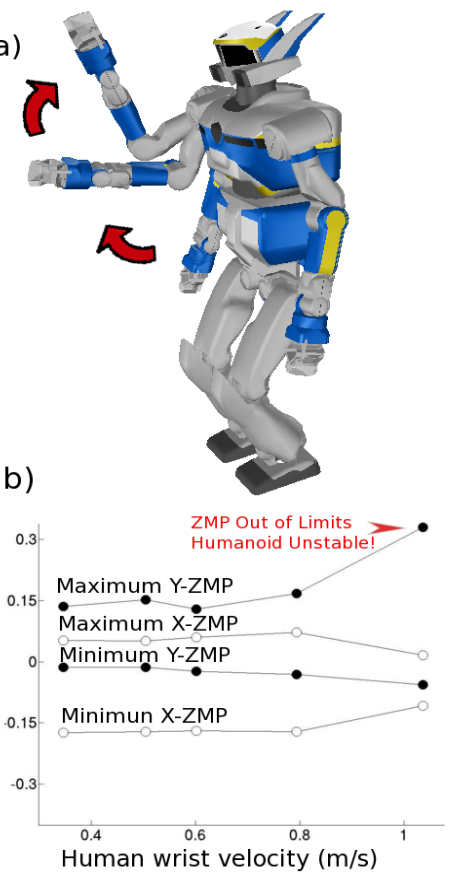

Figure 6: (a) Illustration of the hand up and down motion on the simulated HRP2, (b)Plot of ZMP of the humanoid robot vs. human hand velocities. Illustrated is the point at which the humanoid becomes unstable because of excessive hand speeds.

way to optimize quasi-static humanoid motion, such that it can be performed faster, but also within the dynamic limits of the humanoid. We recorded the online dancing motion of the humanoid, and optimized it offline to see how much faster the same motion could be played. We found that our original 60 seconds of dancing motion could be optimized to 31 seconds while respecting the kinematic and dynamic limits of the humanoid HRP2. Thus, offline optimization could serve as a benchmark in judging how effective and robust online motion retargeting algorithms really are.

Finally, one limitation of our formulation of the HN model is that it cannot guarantee self-collison avoidance on the humanoid. This could be included at the inverse kinematics stage by defining self-collison avoidance as a constraint (Dariush et al., 2008a) (Kanoun, 2009) (Kanehiro et al., 2008a).

\section{SUMMARY AND FUTURE PERSPECTIVES}

In this study we have presented an online method by which a humanoid robot can imitate human motion. The evaluation of the results show that the mo- tion generated by the humanoid closely resembles the original human motion. The proposed CoM anticipation model allows the humanoid to balance itself on one foot taking the cue from the human. This model inspired from neuroscience research opens up new windows towards incorporating biological principles in humanoid motion control (Berthoz, 2000), (Sreenivasa et al., 2009). The use of the HumanoidNormalized model allows standardization across anthropomorphic figures irrespective of proportions (for example marionettes). To further improve imitation it could be interesting to consider the exact dynamics of the humanoid, as well as self-collison avoidance, in the motion planning algorithm. At the present state of this work, we can say that our humanoid robot HRP2 , is capable of mimicking Tai-chi like movements, but not yet quite at the level of Karate.

\section{ACKNOWLEDGEMENTS}

F.J. Montecillo-Puente benefits of a Mexican CONACyT, SEP grant. The authors would like to thank Anthony Mallet, Tan Viet Anh Truong and Oussama Kanoun for taking part in helpful discussions and assisting with the experiments on HRP2 and motion capture. Part of this work is supported by the French ANR Project Locanthrope.

\section{REFERENCES}

Berthoz, A. (2000). The brain's sense of movement. Harvard University Press, Cambridge, MA.

Boulic, R., Maupu, D., and Thalmann, D. (2009). On scaling strategies for the full body interaction with virtual mannequins. Journal Interacting with Computers, Special Issue on Enactive Interfaces, 21(1-2):11-25.

Chois, K. J. and Ko, H. S. (December 2000). Online motion retargetting. The Journal of Visualization and Computer Animation, 11(5):223-235.

Dariush, B., Gienger, M., Arumbakkam, A., Goerick, C., Zhu, Y., and Fujimura, K. (2008a). Online and markerless motion retargeting with kinematic constraints. In IEEE/RSJ International Conference on Intelligent Robots and Systems, pages 191-198.

Dariush, B., Gienger, M., Jian, B., Goerick, C., and Fujimura, K. (2008b). Whole body humanoid control from human motion descriptors. In IEEE International Conference on Robotics and Automation, pages 2677-2684.

Fleury, S., Herrb, M., and Chatila, R. (1997). Genom: A tool for the specification and the implementation of operating modules in a distributed robot architecture. In IEEE/RSJ International Conference on Intelligent Robots and Systems, pages 842-848. 
Kanehiro, F., Lamiraux, F., Kanoun, O., Yoshida, E., and Laumond, J.-P. (2008a). A local collision avoidance method for non-strictly convex polyhedra. Robotics: Science and Systems, IV.

Kanehiro, F., Suleiman, W., Lamiraux, F., Yoshida, E., and Laumond, J.-P. (2008b). Integrating dynamics into motion planning for humanoid robots. In IEEE/RSJ International Conference on Intelligent Robots and Systems, pages 660-667.

Kanoun, O. (2009). Task-driven motion control for humanoid robots. $\mathrm{PhD}$ thesis, LAAS-CNRS; Université de Toulouse.

Multon, F., Kulpa, R., and Bideau, B. (2008). Mkm: A global framework for animating humans in virtual reality applications. Presence: Teleoper. Virtual Environ., 17(1):17-28.

Nakamura, Y. (1991). Advanced Robotics: Redundancy and Optimization. Addison-Wesley Longman Publishing, Boston.

Nakaoka, S., Nakazawa, A., Kanehiro, F., Kaneko, K., Morisawa, M., and Ikeuchi, K. (2005a). Task model of lower body motion for a biped humanoid robot to imitate human dances. In IEEE/RSJ International Conference on Intelligent Robots and Systems, pages 3157-3162.

Nakaoka, S., Nakazawa, A., Kanehiro, F., Kaneko, K., Morisawa, M., and Ikeuchi, K. (2005b). Task model of lower body motion for a biped humanoid robot to imitate human dances. In IEEE/RSJ International Conference on Intelligent Robots and Systems, pages 3157-3162.

Patla, A., Adkin, A., and Ballard, T. (1999). Online steering: coordination and control of body center of mass, head and body reorientation. Experimental Brain Research, 129(4):629-634.

Ruchanurucks, M., Nakaoka, S., Kudoh, S., and Ikeuchi, K. (2006). Humanoid robot motion generation with sequential physical constraints. In IEEE International Conference on Robotics and Automation, pages 2649-2654.

Schaal, S., Ijspeert, A., and Billard, A. (2003). Computational Approaches to Motor Learning by Imitation. philosophical transactions: biological sciences, 358(1431):537-547. philosophical transactions: biological sciences (The Royal Society).

Shon, A., Grochow, K., and Rao, R. (2005). Robotic imitation from human motion capture using gaussian processes. In 5th IEEE-RAS International Conference on Humanoid Robots.

Siciliano, B. and Slotine, J. (1991). A general framework for managing multiple tasks in highly redundant robotic systems. In IEEE Internatioal Conference on Advanced Robotics, pages 1211-1216.

Sreenivasa, M.-N., Soueres, P., Laumond, J.-P., and Berthoz, A. (2009). Steering a humanoid robot by its head. In IEEE/RSJ International Conference on Intelligent Robots and Systems.

Suleiman, W., Yoshida, E., Kanehiro, E., Laumond, J.-P., and Monin, A. (2008). On human motion imitation by humanoid robot. In IEEE International Conference on Robotics and Automation.

Takano, W., Yamane, K., and Nakamura, Y. (2007). Capture database through symbolization, recognition and generation of motion patterns. In IEEE International Conference on Robotics and Automation, pages 3092 3097.

Ude, A., Atkeson, C., and M., R. (2004). Programming full-body movements for humanoid robots by observation. In Robotics and Autonomous Systems, volume 47, pages 93-108.

Vallis, L. and McFadyen, B. (2005). Children use different anticipatory control strategies than adults to circumvent an obstacle in the travel path. Experimental Brain Research, 167(1):119-127.

Vukobratovic, M. and Stepanenko, J. (1972). On the stability of anthropomorphic systems. Mathematical Biosciences, 15:1-37.

Yamane, K. and Hodgins, J. (2009). Simultaneous tracking and balancing of humanoid robots for imitating human motion capture data. In IEEE/RSJ International Conference on Intelligent Robots and Systems, pages 2510-2517.

Yoshida, E., Kanoun, O., Esteves, C., and Laumond, J.P. (2006). Task-driven support polygon humanoids. In IEEE-RAS International Conference on Humanoid Robots. 